\title{
EDITORIAL
}

\section{Greek Gods and Portuguese Pneumology: implementing HERMES}

The HERMES initiative (Harmonising Education in Respiratory Medicine for European Specialists), was created by the European Respiratory Society in 2005, and named after the Greek god of travellers and boundaries. The main goal is the European harmonisation of post-graduate education and training in respiratory medicine, promoting excellence of care and professional mobility. The development strategy involved the creation of a European syllabus and curriculum, method of assessment (establishing a European diploma), and the accreditation of training centres. Besides adult respiratory medicine, HERMES includes education projects on paediatric respiratory medicine, sleep medicine, physiotherapy, critical care, respiratory infections, thoracic oncology, thoracic surgery and spirometry.

The Portuguese post-graduate medical training system is well structured, with nationwide curriculum, accreditation of training centres and final board examination. Our Board, Scientific Society and trainees have already shown commitment to the HERMES programme. As of July of 2015, the public HERMES Diplomates registry includes 342 physicians, of which 25 are Portuguese (22 in adult and 3 in paediatric respiratory medicine).

The discussion, knowledge and tools already produced under the HERMES programme creates an opportunity for reform and improvement of Portuguese training that should not be missed. A collaboration between the Portuguese Respiratory Society (SPP), Medical Association (Ordem dos Médicos) and HERMES programme is already underway. In March of 2015, during the 2015 Lung Science Conference in Estoril, these institutions sat together and formed a small team, including the SPP president (Carlos Robalo Cordeiro), SPP secretary (Sofia Santos), the President of the Pneumology Board (Fernando Barata) and another member of the Board Tiago Alfaro.

This team will be working on further integration of the HERMES initiative on the national training system, including official recognition of the European Examination as an "in training" assessment, and as part of the final residency assessment. The first step was already taken when the European Examination was held for the $5^{\text {th }}$ year Portuguese residents last January in Lisbon, as an "in training assessment'. The accreditation of training centres, using widely discussed criteria can be an important tool for improving the quality of our Residency programmes. A revised criteria document for the accreditation of training centres in adult respiratory medicine and criteria related to paediatric respiratory medicine will be published in 2016. Finally, the recent creation of the Associação Respiratória de Língua Portuguesa (ARELP), an international association of Portuguese speaking Respiratory Medicine Societies and Organisations will pave the way. ARELP's mission will be to promote lung health through the coordination of the collective knowledge, expertise, and resources of its constituting societies in the Portuguese Language Areas worldwide. In this context, and should there be sufficient demand worldwide, the ERS will develop a jointly-funded programme with SSP for the adaptation and translation of its HERMES programme into Portuguese. This is a significant task that will bring tremendous gains in access to high quality learning sources for Portuguese speaking respiratory physicians in Europe, Africa and Brazil.

The official agreement will be signed during SPP national congress in November in Praia da Falésia by the leaders of SPP, SBPT (Brazilian Society of Pneumology and Phthisiology), ERS, ALAT (Latin American Thoracic Association) and representatives of Angola, Mozambique and Cape Vert. An important HERMES activity on spirometry, Part 1 of the ERS spirometry training programme course will already take place during this congress.

In conclusion, now is the moment for further implementation of HERMES in Portuguese Pneumology, following the ERS Strategy principles. ${ }^{1}$ The reforms are already in motion, and the advances will cross continents.

We are confident in these pathways for sharing and increasing education and knowledge in Respiratory Medicine! 


\section{Reference}

1. Migliori GB, Rabe KF, Bel E, et al. The European Respiratory Soci ety plans its future: the 2013-2018 strategic plan. Eur Respir J. 2014;43:927-32.

T. Alfaro $^{a}$, F. Barata ${ }^{a}$, M. Elphinstone ${ }^{b}$, S. Sutter ${ }^{c}$, S. Santos ${ }^{d}$, S. Mitchell ${ }^{b}$, R. Maciel ${ }^{e}$, A Casas $^{f}{ }^{f}$, B. Migliori ${ }^{\mathrm{g}}$, C Robalo Cordeiro ${ }^{\mathrm{h}, *}$

a Portuguese National Board of Pneumology

${ }^{b}$ European Respiratory Society, HERMES Programme c European Board for Accreditation in Pneumology, Senior Coordinator d Portuguese Society of Pneumology, Secretariat e Brazilian Society of Pneumology and Phthisiology,

President

${ }^{f}$ Latin American Thoracic Association, President ${ }^{g}$ European Respiratory Society, Secretary General h Portuguese Society of Pneumology, President

* Corresponding author. Clínica Universitária de Pneumologia, Centro Hospitalar e Universitário de Coimbra, 3000 Coimbra, Portugal. E-mail address: carlos.crobalo@gmail.com

(C.R. Cordeiro). 